\title{
OPTE special issue on PDE-constrained optimization
}

\author{
Michael Ulbrich ${ }^{1} \cdot$ Boris Vexler ${ }^{2}$
}

Received: 10 September 2021 / Revised: 10 September 2021 / Accepted: 10 September 2021 /

Published online: 7 October 2021

(c) The Author(s) 2021

\section{Introduction}

The accurate modeling of complex physical and technical systems relies heavily on PDEs. The resulting systems live in infinite-dimensional function spaces and can involve nonlinearity, nonsmoothness, or uncertainty. Optimization with PDE constraints is the enabling discipline for analyzing and solving highly important problem classes connected to these systems, such as: shape and topology optimization, optimal control, inverse problems, parameter identification, etc. Beyond more traditional applications, the field is increasingly interacting with other timely and important areas, such as uncertainty quantification, data science, or mathematical imaging. Studying the theoretical and numerical aspects of PDE-constrained optimization problems in their original function space setting and tying the developments closely to the latest theoretical and computational advances for PDEs are key elements for a strong theory and for robust, mesh-independent solvers. Mathematically, PDE-constrained optimization is as rich as its numerous applications: It combines theoretical and practical methodology from optimization, PDEs, functional analysis, nonsmooth and variational analysis, numerical analysis, and scientific computing; it also can involve probability and measure theory. A previous OPTE special issue (Ulbrich and van Bloemen 2018) already highlighted many attractive perspectives of PDE-constrained optimization and the current special issue continues to showcase recent advances in PDE-constrained optimization at the intersection of mathematics and engineering applications.

Michael Ulbrich

mulbrich@ma.tum.de

Boris Vexler

vexler@ma.tum.de

1 Chair of Mathematical Optimization, Department of Mathematics, Technical University of Munich, Boltzmannstr. 3, 85748 Garching, Germany

2 Chair of Optimal Control, Department of Mathematics, Technical University of Munich, Boltzmannstr. 3, 85748 Garching, Germany 


\section{Contributions}

In Brenner et al. (2021), Susanne Brenner, Li-yeng Sun, and Winnifried Wollner develop error estimates for finite element discretizations of linear-quadratic elliptic optimal control problems with pointwise constraints on the derivative of the state. Due to the challenging nature of this type of state constraints, the analysis is done for the case of one space dimension. The Karush-Kuhn-Tucker conditions result in a fourth order variational inequality. Depending on the type of boundary conditions, error estimates for a cubic Hermite finite element discretization are derived. The obtained rates are verified by numerical experiments.

In their contribution (Failer and Richter 2021), Lukas Failer and Thomas Richter present an efficient numerical approach for the optimal control of 2D and 3D unsteady fluid-structure interaction (FSI) problems. The optimization uses a BFGS method with adjoint-based gradient computations. A main focus is on deriving efficient multigrid preconditioned methods for solving the large scale systems that arise in each time step of the discretized FSI problem and its adjoint. The nonlinear system of the state equation is solved by an approximate Newton method where the Jacobian is modified such that it becomes block triangular, thus yielding a decoupling into 3 subsystems. For the adjoint system, which is linear and involves the transpose of the Jacobian, a Richardson method preconditioned by the transpose of the matrix used in the approximate Newton method is used. This also results in 3 decoupled linear subsystem solves per Richardson iteration. All arising subsystems are solved by multigrid preconditioned GMRES. Numerical results for 2D and 3D problems demonstrate the efficiency of the approach.

The paper (Hoppe and Neitzel 2021) by Fabian Hoppe and Ira Neitzel analyzes the fast local convergence of sequential quadratic programming (SQP) methods for the optimal control of quasilinear parabolic PDEs. The quasilinearity poses significant challenges compared to, e.g., semilinear state equations. The convergence analysis uses an approach that avoids the typical two-norm discrepancy in second order sufficient conditions. This allows to formulate all convergence results based on "closeness" with respect to the $L^{2}$-topology instead of the usual approach with $L^{\infty}$-neighborhoods from the literature. Moreover, the authors provide a novel regularity result for the adjoint state based on current maximal parabolic regularity estimates. Numerical examples illustrate the theoretical results.

The paper (Mannel and Rund 2021) by Florian Mannel and Armin Rund combines a quasi-Newton approach with a semismooth Newton method to solve nonsmooth optimal control problems. The cost function is the sum of a smooth part and a closed convex part. Building on optimality conditions that involve the proximal operator of the convex part, a semismooth Newton type method is developed that approximates the Hessian of the smooth part by quasi-Newton updates. Superlinear convergence is proved and the assumptions are verified for bound constrained sparse optimal control of linear and semilinear heat equations. The performance of the method is then demonstrated for the optimal control of the heat equation and for bilinear optimal control of the Bloch equation, in both cases with a nonsmooth sparsity term and subject to control constraints. 
Masoumeh Mohammadi and Winnifried Wollner study in Mohammadi and Wollner (2021) an optimal control problem for linearized fracture propagation. The state of the system consists of a phase field that describes the fracture region and a displacement field. The problem is time-discretized and the control of a single time step is considered. The model contains regularization and penalization terms. A linearization around a reference state is used to make the optimal control problem linearquadratic. Subproblems of this form would, e.g., arise in SQP-type methods applied to the original (nonlinear) problem. Since the operator in the linearized fracture equation is in general not coercive, quantitative error estimates are already challenging for this equation itself. The authors derive first a quasi-best approximation result for the FEM approximation of the state equation. Then, based on this, they develop a priori error analysis for the discretization of the optimal control problem resulting in error estimates for the state, the adjoint, and the control variable. Numerical experiments illustrate reduced convergence rates compared to more standard (smooth) settings.

\section{Funding Open Access funding enabled and organized by Projekt DEAL.}

Open Access This article is licensed under a Creative Commons Attribution 4.0 International License, which permits use, sharing, adaptation, distribution and reproduction in any medium or format, as long as you give appropriate credit to the original author(s) and the source, provide a link to the Creative Commons licence, and indicate if changes were made. The images or other third party material in this article are included in the article's Creative Commons licence, unless indicated otherwise in a credit line to the material. If material is not included in the article's Creative Commons licence and your intended use is not permitted by statutory regulation or exceeds the permitted use, you will need to obtain permission directly from the copyright holder. To view a copy of this licence, visit http://creativecommons.org/licen ses/by/4.0/.

\section{References}

Brenner SC, Sung LY, Wollner W (2021) Finite element methods for one dimensional elliptic distributed optimal control problems with pointwise constraints on the derivative of the state. Optim Eng. https://doi.org/10.1007/s11081-020-09491-1

Failer L, Richter T (2021) A Newton multigrid framework for optimal control of fluid-structure interactions. Optim Eng. https://doi.org/10.1007/s11081-020-09498-8

Hoppe F, Neitzel I (2021) Convergence of the SQP method for quasilinear parabolic optimal control problems. Optim Eng. https://doi.org/10.1007/s11081-020-09547-2

Mannel F, Rund A (2021) A hybrid semismooth quasi-Newton method for nonsmooth optimal control with PDEs. Optim Eng. https://doi.org/10.1007/s11081-020-09523-w

Mohammadi M, Wollner W (2021) A priori error estimates for a linearized fracture control problem. Optim Eng. https://doi.org/10.1007/s11081-020-09574-z

Ulbrich M, van Bloemen WB (2018) An introduction to partial differential equations constrained optimization. Optim Eng 19(3):515-520

Publisher's Note Springer Nature remains neutral with regard to jurisdictional claims in published maps and institutional affiliations. 